\title{
High Resolution Spectrometer Coupling to the Sub-Å IBM STEM
}

\author{
P.E. Batson
}

\section{IBM T.J. Watson Research Center, Yorktown Heights, New York 10598}

With the recent installation of the Nion spherical aberration corrector in the VG Microscopes HB501 STEM at IBM, [1, 2] major changes in the optical coupling between the specimen and spectrometer are needed. In the original configuration, a simple doublet quadrupole was used to image the STEM probe into the deceleration field of the high voltage electrode which houses the Wien Filter spectrometer.[3] Within the electrode, the filter pass energy is 100$150 \mathrm{eV}$, producing a very high energy dispersion of about $100 \mu \mathrm{m} / \mathrm{eV}$. Finally, the dispersed spectrum is imaged by the re-acceleration lens onto a CCD detector with a dispersion of about $1 \mathrm{~mm} / \mathrm{eV}$. For about $70 \mathrm{meV}$ resolution, a CCD using about 300, $25 \mu \mathrm{m}$ wide pixels gives an energy window of 6-7 eV. Wider energy spectra are obtained by scanning the center pass energy of the spectrometer, and subsequently recombining many overlapping energy windows.[4]

After installation of the corrector system, the space previously occupied by the doublet quadrupole has been occupied by the Ronchigram camera. In addition, with the smaller probe size, the angular beam spread has increased to about $25 \mathrm{mR}$ half width. Finally, in this instrument, an electron momochromator is being added to improve the desired EELS energy resolution from $0.2 \mathrm{eV}$, limited by the field emission source, to about $60-80 \mathrm{meV}$.[5] Therefore, the new spectrometer optical coupling needs to be shifted to a new location; it must be capable of handling larger input angles; and it must allow an improved energy resolution in the spectrometer.

Figure 1 gives a summary of the present solution for these requirements. Two planes are shown, corresponding to the dispersive and non-dispersive directions of the Wien filter. Briefly, on the left, the STEM electron beam exits the objective lens with a compression of about 3x, giving a $3 \mathrm{mR}$ half angle for $15 \mathrm{mR}$ at the specimen. It then passes through the ADF detector, and enters a "snorkel" lens, which is situated below the Ronchigram TV camera, and which therefore must have a very large bore. Above the camera, a weak quadrupole shapes the beam for entry into the decelleration field of the Wien Filter. Finally, energy dispersion, re-acceleration and imaging at the CCD are done as in the past. The snorkel design was chosen to minimize spherical aberration given the need for a large bore, long focal length lens. This particular combination has a bore of $20 \mathrm{~mm}$, a focal length of $138 \mathrm{~mm}$ and a spherical aberration coefficient of $19 \mathrm{~m}$. Within the spectrometer electrode, the beam is brought to s $16 \mu \mathrm{m}$ diameter focus in the energy dispersive direction. In the non-dispersive direction, a $9 \mu \mathrm{m}$ diameter parallel beam is achieved - limited by spherical aberration in the snorkel lens. At the CCD, a 180x15 $\mu \mathrm{m}$ line focus is made for use with a new CCD detector having $10 \mu \mathrm{m}$ pixel size. Given the $1 \mathrm{~mm} / \mathrm{eV}$ dispersion, the resolution of this system should be about $15 \mathrm{meV}$ for $15 \mathrm{mR}$ half angle at the specimen. Recent improvement in the electronics 
of the spectrometer should allow an instability imposed limit of about 20-30 meV.

With this instrument, it should be possible to obtain soft x-ray core edges with a resolution limited only by the core hole lifetime (about $100 \mathrm{meV}$ for the $\mathrm{Si} \mathrm{L}_{2,3}$ edge). With a larger collection aperture, a large fraction of the available scattering should be accessible at 0.1$0.2 \mathrm{eV}$ resolution. It may also be able to probe the direct inter-band scattering below $1 \mathrm{eV}$ in semiconductors - an area of extreme interest that has previously been inaccessible to instruments having atomic column resolution.

[1] N. Dellby, O.L. Krivanek, P.D. Nellist, P.E. Batson, and A.R. Lupini, J. Electron Microscopy, 50 (2001)177 $-185$.

[2] P.E. Batson, Niklas Dellby, and O.L. Krivanek, Nature, 418 (2002)617 - 620.

[3] P.E. Batson, Rev. Sci. Inst., 57 (1986)43-48.

[4] P.E. Batson, Rev. Sci. Inst., 59 (1988)1132 - 1138.

[5] H.W. Mook, P.E. Batson, and P. Kruit, in Electron Microscopy and Analysis 1999, edited by C.J. Kiely (Institute of Physics, Bristol, 1999), Vol. 161, pp. 223 - 226.

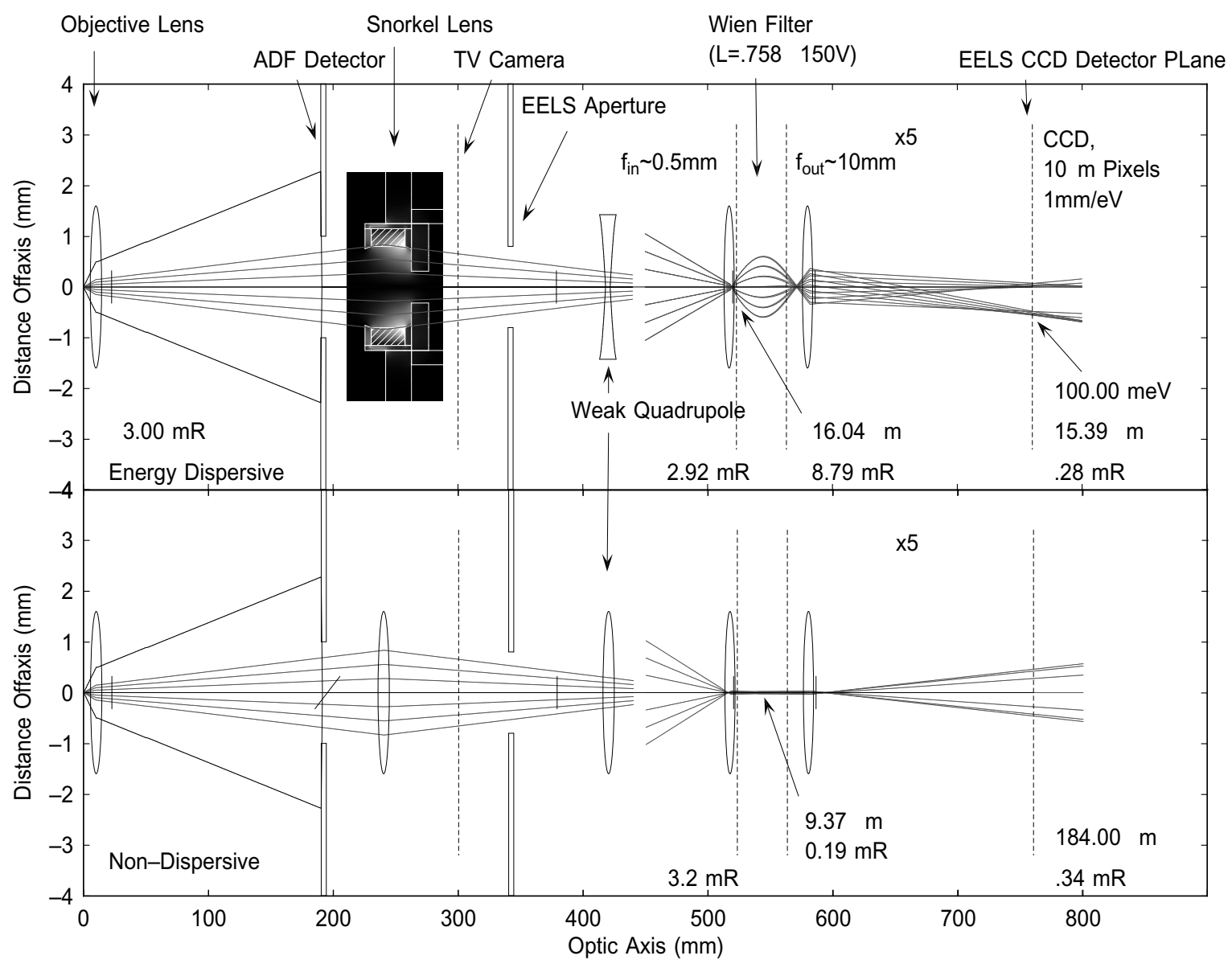

Fig. 1. Optical design for coupling of the Wien filter spectrometer to the specimen in the IBM aberration corrected STEM. 